\title{
Orientation mapping in super-resolution
}

Fluorescence polarization enables superresolution dipole orientation mapping.

In 2014, Peter Walla and colleagues published a method called super-resolution by polarization demodulation (SPoD), in which information on fluorophore orientation is used to overcome the diffraction barrier. In this method, a sample is illuminated with polarized excitation light, and the periodic signals emitted with different phases are detected. These signals are then reconstructed with a deconvolution algorithm (SPEED) to produce super-resolution images.

Further interrogation of SPoD revealed that the SPEED algorithm alone could account for the increased resolution achieved, independent of polarization information, casting doubt on whether polarization modulation was in fact useful for super-resolution microscopy. Walla et al. have argued that polarization information alone can be used for resolution gain.
Nevertheless, to some this remains an open question.

Dayong Jin at the University of Technology Sydney, Juntao Gao at Tsinghua University, Peng Xi at Peking University and colleagues have now built upon SPoD to develop superresolution dipole orientation mapping (SDOM), which can determine and map the orientation of molecules in a super-resolution image. Prior to this method, the orientation of molecules could be mapped only with diffraction-limited resolution.

In SPoD, the dipole orientation is lost during the image-reconstruction process. SDOM bypasses the use of the SPEED algorithm and instead uses fluorophore intensity to generate a super-resolution image. It then extracts phase information to determine dipole orientation, creating a dipole orientation map that can be laid over the image.

To demonstrate the strength of their method, the researchers began by comparing
SPoD and SDOM images of dendritic spine necks in membrane-labeled hippocampal neurons. The SDOM images retained the same level of resolution as those obtained using SPoD, while gaining detailed dipole orientation information. The team went on to show that orientation information could be obtained for actin in fixed mammalian cells; the filaments were highly polarized and could be clearly resolved from one another. Similar results were obtained for septin and a nuclear pore complex protein in live yeast. SDOM represents an exciting method for studying the orientation of biological molecules in super-resolution.

\section{Rita Strack}

\section{RESEARCH PAPERS}

Hafi, N. et al. Fluorescence nanoscopy by polarization modulation and polarization angle narrowing. Nat. Methods 11, 579-584 (2014).

Zhanghao, K. et al. Super-resolution dipole orientation mapping via polarization demodulation. Light: Science \& Applications 5, e16166 (2016). 